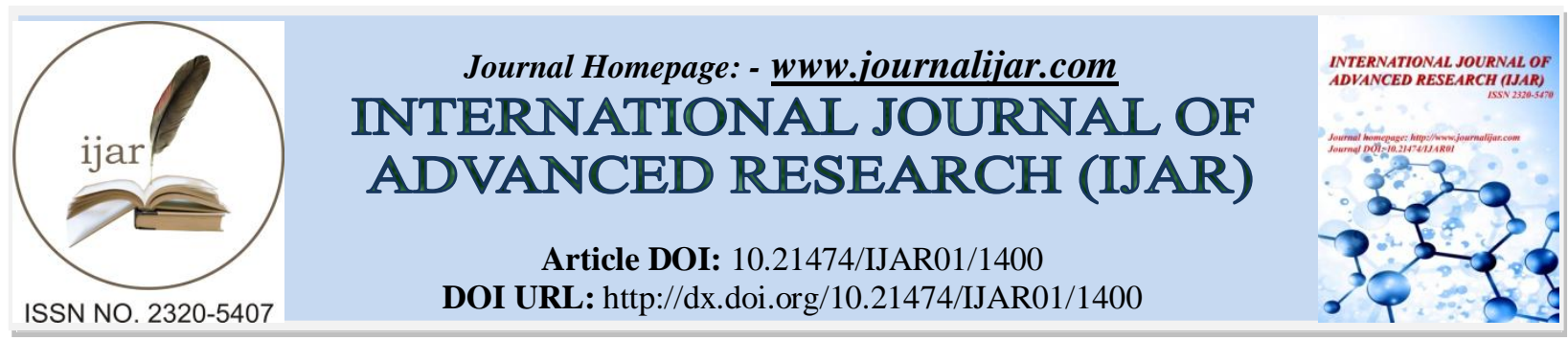

RESEARCH ARTICLE

\title{
PERFORMANCE OF INDIAN BANKING SECTOR WITH REFERENCE TO CAPITAL ADEQUACY RATIO UNDER BASEL NORMS - A STUDY OF SELECTED BANKS.
}

\author{
*Rakesh Kumar. \\ Research Scholar, IKG Punjab Technical University, Kapurthala.
}

\section{Manuscript Info}

Manuscript History

Received: 12 June 2016

Final Accepted: 16 July 2016

Published: August 2016

Key words:-

Performance, Basel Norms, Capital Adequacy Ratio, Public sector Banks, Private Sector Banks, Foreign Sector Banks

\begin{abstract}
The Indian Banking has seen momentous changes in the postindependence era. The operating environment of banks in India has been gradually changing from the last decade. With the adoption of Basel norms, the performance of banks in India is reviewed after taking the capital adequacy ratio of banks selected for study. For this study, five banks from each sector (public sector banks, private sector banks, foreign sector banks) is selected. Performance of banks is measured on the basis of only four criteria selected: Capital Adequacy Ratio, N.P.A., Business per employee and Profit per employee. Overall, the analysis supports the conclusion that foreign owned banks are on average performs better as compare to other sector banks.
\end{abstract}

Copy Right, IJAR, 2016,. All rights reserved.

\section{Introduction:-}

The performance of any economy, to a large extent, is dependent on the performance of the banks. The Indian Banking has seen momentous changes in the post-independence era. The operating environment of banks in India has been gradually changing from the last decade. Various reforms and measures both qualitative and quantitative were introduced with an objective to revitalize the banking sector and enable it to meet the future challenges. Basel norms established taking into account the various changes seen in the banking industry in present environment.

\section{Basel Norms- An Introduction:-}

In 1988, the Basel Committee introduced the first Basel Accord on Minimum Capital Requirement for internationally, active banks, in order to promote sound and stable banking systems and a worldwide level playing field. At present over 100 countries have adopted this capital regulatory framework, often also applying it to locally active banks. Bank's capital ration not fall below 8 percent. This ratio indicates bank's capability to absorb losses. BIS ratio consists of Tier I, Tier II \& Tier III capital. Tier I capital includes Equity Shares, Reserves etc. Tier II consists of preferred shares and debt certificates with no fixed maturity. Tier III consists of short term subordinated debts and accounts for only a small share of actual own funds.

Basel II is the second of the Basel accords, which are recommendations on banking laws and regulations issued by the Basel Committee on Banking Supervision. The purpose of Basel II, which was initially published in June 2004, is to create an international standard that banking regulators can use when creating regulations about how much capital banks need to put aside to guard against the types of financial and operational risks banks face while maintaining sufficient consistency so that this does not become a source of competitive inequality amongst internationally active banks. Advocates of Basel II believe that such an international standard can help protect the 
international financial system from the types of problems that might arise should a major bank or a series of banks collapse.

Basel III is a comprehensive set of reform measures, developed by the Basel Committee on Banking Supervision, to strengthen the regulation, supervision and risk management of the banking sector. These measures aim to:

* improve the banking sector's ability to absorb shocks arising from financial and economic stress, whatever the source

* improve risk management and governance

* Strengthen banks' transparency and disclosures.

* It is expected that Basel III will be implemented progressively across the European Union (and elsewhere) between 2013 and 2019. The implementation will require a series of directives and regulations to be introduced at both Community and national level.

* One of the main outcomes of Basel III will be a significant rise in the banking industry's capital requirements

\section{Objective of the Study:-}

The principal objective of the study is to examine the various aspects relating to the performance of banking industry in India taking into account Capital Adequacy Ratio prescribed under Basel norms and some selected financial indicators.

\section{Research Methodology:-}

Scope of the Study:-

The present study covers an in-depth analysis of the prospects of growth of public private and foreign sector banks in India. The study has to covers the period from 2001-02 to 2014-15. This fourteen years period is sufficient to examine the performance of nationalized banks. The study has also some limitation because research is concerned to selected public, private and foreign sector banks in India. Five banks from each sector selected on the basis of their business volume and other leading indicators from their respective fields.

\section{Collection of Data:-}

The proposed study shall base upon secondary data. The secondary data will be collected from published information by different governmental agencies, such as Reserve Bank of India, IBA Bulletins, different committee's reports and data etc.

\section{Review Of Literature:-}

Sankharaj Roy, Biplab Kumar De (2013), in their research paper titled 'An analysis of Financial Performance of Indian Commercial Banks' studied the various financial aspects of banks in India. The present study was done to examine the importance of financial performance of the commercial banks during the period of 2000 to 2011 . The study applied key profitability ratios for assessing the financial performance of the commercial banks. Financial stability of the banks plays a crucial role in the growth of the banks. To accomplish this objective a regression analysis between Earnings before interest and tax and different factors affecting banks profitability was done. The study reveals that the during the period Return on Assets which indicates how efficiently the company is using its total assets shows an increasing trends in the last five year from 2007 to 2011. Return on Net Worth shows an increasing trend from 2007 onwards with very poor performance in 2006. The Capital adequacy ratio has strong negative relation with Net Assets to RONW ratio and NPA to net assets ratios.

Akshay Uday Shenoy, Yatin Balkrishan Mohane \& Charan Singh (2014) in their working paper on Basel Banking Norms- A PRIMER, understanding of the emergence of Basel banking norms (Basel I), and the transition to each of the subsequent regulations (Basel II and Basel III). The paper suggest that Compliance with Basel norms requires a high level of capital, which brings down the competitiveness of a bank. Adoption of Basel norms in countries having inadequate resources could result in the inadvertent diversion of capital from more vital avenues.

\section{Data Analysis and Findings:-}

In this paper, Performance of banks is based upon only four criteria i.e. Capital Adequacy Ratio, NPA, Business per employee, Profit per employee etc. 
Table 1:- Capital Adequacy Ratio of Banks Selected for Study (in \%)

\begin{tabular}{|c|c|c|c|c|c|c|c|c|c|c|c|c|c|c|}
\hline $\begin{array}{l}\text { Year/Ba } \\
\text { nk }\end{array}$ & $\begin{array}{l}200 \\
1-02\end{array}$ & $\begin{array}{l}200 \\
2-03\end{array}$ & $\begin{array}{l}200 \\
3-04\end{array}$ & $\begin{array}{l}200 \\
4-05\end{array}$ & $\begin{array}{l}200 \\
5-06\end{array}$ & $\begin{array}{l}200 \\
6-07\end{array}$ & $\begin{array}{l}200 \\
7-08\end{array}$ & $\begin{array}{l}200 \\
8-09\end{array}$ & $\begin{array}{l}200 \\
9-10\end{array}$ & $\begin{array}{l}201 \\
0-11\end{array}$ & $\begin{array}{l}201 \\
1-12\end{array}$ & $\begin{array}{l}201 \\
2-13\end{array}$ & $\begin{array}{l}201 \\
3-14\end{array}$ & $\begin{array}{l}201 \\
4-15\end{array}$ \\
\hline SBI & $\begin{array}{l}13.3 \\
5\end{array}$ & $\begin{array}{l}13.5 \\
0\end{array}$ & $\begin{array}{l}13.5 \\
3\end{array}$ & $\begin{array}{l}12.4 \\
5\end{array}$ & $\begin{array}{l}11.8 \\
8\end{array}$ & $\begin{array}{l}12.3 \\
4\end{array}$ & $\begin{array}{l}13.5 \\
4\end{array}$ & $\begin{array}{l}14.2 \\
5\end{array}$ & $\begin{array}{l}13.3 \\
9\end{array}$ & $\begin{array}{l}11.9 \\
8\end{array}$ & $\begin{array}{l}13.8 \\
6\end{array}$ & $\begin{array}{l}12.9 \\
2\end{array}$ & $\begin{array}{l}12.4 \\
4\end{array}$ & $\begin{array}{l}12.0 \\
0\end{array}$ \\
\hline PNB & $\begin{array}{l}10.7 \\
0\end{array}$ & $\begin{array}{l}12.0 \\
2 \\
\end{array}$ & $\begin{array}{l}13.1 \\
0 \\
\end{array}$ & $\begin{array}{l}14.7 \\
8\end{array}$ & $\begin{array}{l}11.9 \\
5\end{array}$ & $\begin{array}{l}12.2 \\
9 \\
\end{array}$ & $\begin{array}{l}13.4 \\
6 \\
\end{array}$ & $\begin{array}{l}14.0 \\
3 \\
\end{array}$ & $\begin{array}{l}14.1 \\
6 \\
\end{array}$ & $\begin{array}{l}12.4 \\
2 \\
\end{array}$ & $\begin{array}{l}12.6 \\
3 \\
\end{array}$ & $\begin{array}{l}12.7 \\
2 \\
\end{array}$ & $\begin{array}{l}11.5 \\
2 \\
\end{array}$ & $\begin{array}{l}12.2 \\
1 \\
\end{array}$ \\
\hline BOB & $\begin{array}{l}11.3 \\
2\end{array}$ & $\begin{array}{l}12.6 \\
5\end{array}$ & $\begin{array}{l}13.9 \\
1\end{array}$ & $\begin{array}{l}12.6 \\
1\end{array}$ & $\begin{array}{l}13.6 \\
5\end{array}$ & $\begin{array}{l}11.8 \\
0\end{array}$ & $\begin{array}{l}12.9 \\
4\end{array}$ & $\begin{array}{l}14.0 \\
5\end{array}$ & $\begin{array}{l}14.3 \\
6\end{array}$ & $\begin{array}{l}14.5 \\
2\end{array}$ & $\begin{array}{l}14.6 \\
7\end{array}$ & $\begin{array}{l}13.3 \\
0\end{array}$ & $\begin{array}{l}12.2 \\
8\end{array}$ & $\begin{array}{l}12.6 \\
1 \\
\end{array}$ \\
\hline $\begin{array}{l}\text { Canara } \\
\text { Bank }\end{array}$ & $\begin{array}{l}11.8 \\
8\end{array}$ & $\begin{array}{l}12.5 \\
0\end{array}$ & $\begin{array}{l}12.6 \\
6 \\
\end{array}$ & $\begin{array}{l}12.7 \\
8\end{array}$ & $\begin{array}{l}11.2 \\
2\end{array}$ & $\begin{array}{l}13.5 \\
0\end{array}$ & $\begin{array}{l}13.2 \\
5\end{array}$ & $\begin{array}{l}14.1 \\
0\end{array}$ & $\begin{array}{l}13.4 \\
3\end{array}$ & $\begin{array}{l}15.3 \\
8\end{array}$ & $\begin{array}{l}13.7 \\
6\end{array}$ & $\begin{array}{l}12.4 \\
0\end{array}$ & $\begin{array}{l}10.6 \\
3\end{array}$ & $\begin{array}{l}10.5 \\
6\end{array}$ \\
\hline $\begin{array}{l}\text { Bank of } \\
\text { India }\end{array}$ & $\begin{array}{l}10.6 \\
8\end{array}$ & $\begin{array}{l}12.0 \\
2 \\
\end{array}$ & $\begin{array}{l}13.0 \\
1\end{array}$ & $\begin{array}{l}11.5 \\
2 \\
\end{array}$ & $\begin{array}{l}10.7 \\
5 \\
\end{array}$ & $\begin{array}{l}11.7 \\
5\end{array}$ & $\begin{array}{l}12.0 \\
4 \\
\end{array}$ & $\begin{array}{l}13.0 \\
1 \\
\end{array}$ & $\begin{array}{l}12.9 \\
4\end{array}$ & $\begin{array}{l}12.1 \\
7 \\
\end{array}$ & $\begin{array}{l}11.9 \\
5\end{array}$ & $\begin{array}{l}11.0 \\
2 \\
\end{array}$ & 9.97 & $\begin{array}{l}10.7 \\
3\end{array}$ \\
\hline ICICI & $\begin{array}{l}11.4 \\
4\end{array}$ & $\begin{array}{l}11.1 \\
0\end{array}$ & $\begin{array}{l}10.3 \\
6\end{array}$ & $\begin{array}{l}11.7 \\
8\end{array}$ & $\begin{array}{l}13.3 \\
5\end{array}$ & $\begin{array}{l}11.6 \\
9\end{array}$ & $\begin{array}{l}13.9 \\
7\end{array}$ & $\begin{array}{l}15.5 \\
3\end{array}$ & $\begin{array}{l}19.4 \\
1\end{array}$ & $\begin{array}{l}19.5 \\
4\end{array}$ & $\begin{array}{l}18.5 \\
2\end{array}$ & $\begin{array}{l}18.7 \\
4\end{array}$ & $\begin{array}{l}17.7 \\
0\end{array}$ & $\begin{array}{l}17.0 \\
2\end{array}$ \\
\hline HDFC & $\begin{array}{l}13.9 \\
3\end{array}$ & $\begin{array}{l}11.1 \\
2\end{array}$ & $\begin{array}{l}11.6 \\
6\end{array}$ & $\begin{array}{l}12.1 \\
6\end{array}$ & $\begin{array}{l}11.4 \\
1\end{array}$ & $\begin{array}{l}13.0 \\
8\end{array}$ & $\begin{array}{l}13.6 \\
0\end{array}$ & $\begin{array}{l}15.6 \\
9\end{array}$ & $\begin{array}{l}17.4 \\
4\end{array}$ & $\begin{array}{l}16.2 \\
2\end{array}$ & $\begin{array}{l}16.5 \\
2\end{array}$ & $\begin{array}{l}16.8 \\
0\end{array}$ & $\begin{array}{l}16.0 \\
7\end{array}$ & $\begin{array}{l}16.7 \\
9\end{array}$ \\
\hline AXIS & $\begin{array}{l}10.6 \\
5\end{array}$ & $\begin{array}{l}10.9 \\
0\end{array}$ & $\begin{array}{l}11.2 \\
1\end{array}$ & $\begin{array}{l}12.6 \\
6\end{array}$ & $\begin{array}{l}11.0 \\
8\end{array}$ & $\begin{array}{l}11.5 \\
7\end{array}$ & $\begin{array}{l}13.7 \\
3\end{array}$ & $\begin{array}{l}13.6 \\
9\end{array}$ & $\begin{array}{l}15.8 \\
0\end{array}$ & $\begin{array}{l}12.6 \\
5\end{array}$ & $\begin{array}{l}13.6 \\
6\end{array}$ & $\begin{array}{l}17.0 \\
0\end{array}$ & $\begin{array}{l}16.0 \\
7\end{array}$ & $\begin{array}{l}12.0 \\
7\end{array}$ \\
\hline $\begin{array}{l}\text { Dhanlax } \\
\text { mi Bank }\end{array}$ & $\begin{array}{l}11.2 \\
3\end{array}$ & $\begin{array}{l}10.4 \\
5 \\
\end{array}$ & $\begin{array}{l}13.5 \\
6 \\
\end{array}$ & $\begin{array}{l}10.1 \\
6 \\
\end{array}$ & 9.75 & 9.77 & 9.21 & $\begin{array}{l}15.3 \\
8 \\
\end{array}$ & $\begin{array}{l}12.9 \\
9 \\
\end{array}$ & $\begin{array}{l}11.8 \\
0\end{array}$ & 9.49 & $\begin{array}{l}11.0 \\
6 \\
\end{array}$ & 8.67 & 9.59 \\
\hline $\begin{array}{l}\text { Federal } \\
\text { Bank }\end{array}$ & $\begin{array}{l}10.6 \\
3\end{array}$ & $\begin{array}{l}11.2 \\
3\end{array}$ & $\begin{array}{l}11.4 \\
8\end{array}$ & $\begin{array}{l}11.2 \\
7\end{array}$ & $\begin{array}{l}13.7 \\
5\end{array}$ & $\begin{array}{l}13.4 \\
3\end{array}$ & $\begin{array}{l}22.4 \\
6\end{array}$ & $\begin{array}{l}20.2 \\
2\end{array}$ & $\begin{array}{l}18.3 \\
6\end{array}$ & $\begin{array}{l}16.7 \\
9\end{array}$ & $\begin{array}{l}16.6 \\
4\end{array}$ & $\begin{array}{l}14.7 \\
3\end{array}$ & $\begin{array}{l}15.1 \\
4\end{array}$ & $\begin{array}{l}15.4 \\
6\end{array}$ \\
\hline $\begin{array}{l}\text { CITI } \\
\text { Bank }\end{array}$ & $\begin{array}{l}11.0 \\
4\end{array}$ & $\begin{array}{l}11.3 \\
0\end{array}$ & $\begin{array}{l}11.1 \\
1\end{array}$ & $\begin{array}{l}10.7 \\
8\end{array}$ & $\begin{array}{l}11.3 \\
3\end{array}$ & $\begin{array}{l}11.0 \\
6\end{array}$ & $\begin{array}{l}12.0 \\
0\end{array}$ & $\begin{array}{l}13.2 \\
3\end{array}$ & $\begin{array}{l}18.1 \\
4\end{array}$ & $\begin{array}{l}17.3 \\
1\end{array}$ & $\begin{array}{l}16.0 \\
3\end{array}$ & $\begin{array}{l}15.9 \\
0\end{array}$ & $\begin{array}{l}16.4 \\
9\end{array}$ & $\begin{array}{l}15.3 \\
0\end{array}$ \\
\hline $\begin{array}{l}\text { Abu } \\
\text { Dhabi }\end{array}$ & $\begin{array}{l}10.4 \\
2 \\
\end{array}$ & $\begin{array}{l}10.1 \\
4 \\
\end{array}$ & $\begin{array}{l}14.2 \\
2 \\
\end{array}$ & $\begin{array}{l}14.3 \\
8\end{array}$ & $\begin{array}{l}36.9 \\
8\end{array}$ & $\begin{array}{l}27.6 \\
6\end{array}$ & $\begin{array}{l}51.7 \\
1\end{array}$ & $\begin{array}{l}47.5 \\
7 \\
\end{array}$ & $\begin{array}{l}44.7 \\
9\end{array}$ & $\begin{array}{l}45.2 \\
5\end{array}$ & $\begin{array}{l}80.8 \\
8\end{array}$ & $\begin{array}{l}66.8 \\
2\end{array}$ & $\begin{array}{l}35.9 \\
2\end{array}$ & $\begin{array}{l}27.5 \\
6\end{array}$ \\
\hline $\begin{array}{l}\text { Bank of } \\
\text { America }\end{array}$ & $\begin{array}{l}21.0 \\
7 \\
\end{array}$ & $\begin{array}{l}21.0 \\
8 \\
\end{array}$ & $\begin{array}{l}22.9 \\
2 \\
\end{array}$ & $\begin{array}{l}30.0 \\
7 \\
\end{array}$ & $\begin{array}{l}23.4 \\
0 \\
\end{array}$ & $\begin{array}{l}13.3 \\
3 \\
\end{array}$ & $\begin{array}{l}13.4 \\
5 \\
\end{array}$ & $\begin{array}{l}12.7 \\
3 \\
\end{array}$ & $\begin{array}{l}15.4 \\
9 \\
\end{array}$ & $\begin{array}{l}14.5 \\
1 \\
\end{array}$ & $\begin{array}{l}17.5 \\
9\end{array}$ & $\begin{array}{l}18.4 \\
0\end{array}$ & $\begin{array}{l}16.7 \\
0\end{array}$ & $\begin{array}{l}15.1 \\
6 \\
\end{array}$ \\
\hline $\begin{array}{l}\text { Standard } \\
\text { Chartere } \\
\text { d Bank }\end{array}$ & 9.28 & $\begin{array}{l}10.5 \\
6\end{array}$ & $\begin{array}{l}10.8 \\
7\end{array}$ & $\begin{array}{l}10.4 \\
6\end{array}$ & 9.93 & $\begin{array}{l}10.4 \\
4\end{array}$ & & $\begin{array}{l}11.5 \\
5\end{array}$ & $\begin{array}{l}12.4 \\
1\end{array}$ & $\begin{array}{l}11.8 \\
8\end{array}$ & $\begin{array}{l}11.0 \\
5\end{array}$ & & $\begin{array}{l}12.4 \\
8\end{array}$ & $\begin{array}{l}12.4 \\
9\end{array}$ \\
\hline HSBC & $\begin{array}{l}10.9 \\
2\end{array}$ & $\begin{array}{l}18.1 \\
0 \\
\end{array}$ & $\begin{array}{l}14.5 \\
4\end{array}$ & $\begin{array}{l}14.0 \\
3 \\
\end{array}$ & $\begin{array}{l}10.6 \\
1 \\
\end{array}$ & $\begin{array}{l}11.0 \\
6\end{array}$ & $\begin{array}{l}10.5 \\
9\end{array}$ & $\begin{array}{l}15.3 \\
1 \\
\end{array}$ & $\begin{array}{l}18.0 \\
3\end{array}$ & $\begin{array}{l}18.0 \\
3 \\
\end{array}$ & $\begin{array}{l}16.0 \\
4\end{array}$ & $\begin{array}{l}17.1 \\
0\end{array}$ & $\begin{array}{l}17.3 \\
6\end{array}$ & $\begin{array}{l}14.8 \\
4 \\
\end{array}$ \\
\hline
\end{tabular}

(Source: RBI and IBA)

In this table, it clearly indicates that in Public sector Banks, most of the banks have maintained appropriate level of CAR but showing decline rate except PNB. In Public sector bank, all the banks maintain high CAR above the prescribed level except Dhanlaxmi Bank which shows declining trends. Yet in Foreign Sector Banks, CAR almost higher than all other sector banks. Overall it has been concluded that foreign sector banks working in India maintain higher capital adequacy and they have also made high level of provisioning to meet the future risk. The percentage increase is higher in foreign sector banks.

Table 2:- Net NPA Ratio of Banks Selected for Study (in \%)

\begin{tabular}{|l|l|l|l|l|l|l|l|l|l|l|l|l|l|l|}
\hline $\begin{array}{l}\text { Year/Ba } \\
\text { nk }\end{array}$ & 200 & 200 & 200 & 200 & 200 & 200 & 200 & 200 & 200 & 201 & 201 & 201 & 201 & 201 \\
\hline SBI & 5.63 & 4.50 & $3-48$ & $4-05$ & $5-06$ & $6-07$ & $7-08$ & $8-09$ & $9-10$ & $0-11$ & $1-12$ & $2-13$ & $3-14$ & $4-15$ \\
\hline PNB & 5.32 & 3.86 & 0.98 & 0.20 & 0.29 & 0.76 & 0.64 & 0.17 & 0.53 & 0.85 & 1.52 & 2.35 & 2.57 & 2.12 \\
\hline BOB & 4.98 & 3.72 & 2.99 & 1.45 & 0.87 & 0.60 & 0.47 & 0.31 & 0.34 & 0.35 & 0.54 & 1.28 & 1.52 & 1.89 \\
\hline $\begin{array}{l}\text { Canara } \\
\text { Bank }\end{array}$ & 3.89 & 3.59 & 2.89 & 1.88 & 1.12 & 0.94 & 0.84 & 1.09 & 1.06 & 1.10 & 1.46 & 2.18 & 1.98 & 2.65 \\
\hline $\begin{array}{l}\text { Bank of } \\
\text { India }\end{array}$ & 6.02 & 5.37 & 4.50 & 2.77 & 1.49 & 0.95 & 0.52 & 0.44 & 1.31 & 0.91 & 1.47 & 2.06 & 2.00 & 3.36 \\
\hline ICICI & 5.48 & 5.21 & 2.21 & 1.65 & 0.72 & 1.02 & 1.55 & 2.09 & 2.12 & 1.11 & 0.73 & 0.77 & 0.97 & 1.61 \\
\hline HDFC & 0.50 & 0.37 & 0.16 & 0.24 & 0.44 & 0.43 & 0.47 & 0.63 & 0.31 & 0.19 & 0.18 & 0.20 & 0.27 & 0.20 \\
\hline
\end{tabular}




\begin{tabular}{|l|l|l|l|l|l|l|l|l|l|l|l|l|l|l|}
\hline AXIS & 3.46 & 2.39 & 1.29 & 1.39 & 0.98 & 0.72 & 0.42 & 0.40 & 0.40 & 0.29 & 0.27 & 0.36 & 0.44 & 0.46 \\
\hline $\begin{array}{l}\text { Dhanlax } \\
\text { mi Bank }\end{array}$ & $\begin{array}{l}11.6 \\
6\end{array}$ & 9.25 & 6.68 & 3.92 & 2.82 & 1.75 & 0.88 & 0.88 & 0.84 & 0.30 & 0.66 & 3.36 & 3.80 & 3.29 \\
\hline $\begin{array}{l}\text { Federal } \\
\text { Bank }\end{array}$ & 8.60 & 4.95 & 2.89 & 2.21 & 0.95 & 0.44 & 0.23 & 0.30 & 0.48 & 0.60 & 0.53 & 0.98 & 0.74 & 0.73 \\
\hline $\begin{array}{l}\text { CITI } \\
\text { Bank }\end{array}$ & 0.40 & 1.17 & 1.40 & 1.00 & 0.95 & 1.02 & 1.23 & 2.63 & 2.14 & 1.21 & 0.90 & 1.47 & 1.24 & 0.40 \\
\hline $\begin{array}{l}\text { Abu } \\
\text { Dhabi }\end{array}$ & 3 & 9.48 & 27.3 & 12.7 & 15.9 & 0.63 & 0.00 & 0.00 & 0.19 & 2.89 & 0.00 & 0.00 & N/A & N/A \\
\hline $\begin{array}{l}\text { Bank of } \\
\text { America }\end{array}$ & 0.80 & 0.05 & 0.00 & 0.00 & 0.00 & 0.00 & 0.00 & 0.00 & 0.00 & 0.00 & 0.00 & 0.00 & 0.00 & 0.11 \\
\hline $\begin{array}{l}\text { Standard } \\
\text { Chartere } \\
\text { d Bank }\end{array}$ & 0.40 & 0.31 & 0.52 & 1.12 & 1.57 & 1.43 & 1.04 & 1.37 & 1.40 & 0.27 & 0.70 & 1.63 & 0.45 & 0.34 \\
\hline HSBC & 2.27 & 1.03 & 0.70 & 0.50 & 0.58 & 0.43 & 0.58 & 1.42 & 2.31 & 0.91 & 0.62 & 0.33 & 0.24 & 0.51 \\
\hline
\end{tabular}

(Source: RBI and IBA)

The net NPA to loans (advances) ratio is used as a measure of the overall quality of the bank's loan book. Higher the ratio indicates that marginal position of the banks regarding loan. In the above said table NPA ratio showing declining trends in the period of study of the entire banking sector. But in foreign sector banks, Some banks such as Bank of America, Abu Dhabi bank maintain near about Zero ratio. This indicates that the recovery of loan in these banks does not create any problem. This indicates the sound business policies, better provisioning of foreign sector banks as compare to other sector banks.

Table 3:- Business per employee of Banks selected for study (IN Rs. Lakhs)

\begin{tabular}{|c|c|c|c|c|c|c|c|c|c|c|c|c|c|c|}
\hline Year/ & 2001 & 2002 & 2003 & 2004 & 2005 & 2006 & 2007 & 2008 & 2009 & 2010 & 2011 & 2012 & 2013 & 2014 \\
\hline Bank & -02 & -03 & -04 & -05 & -06 & -07 & -08 & -09 & -10 & -11 & -12 & -13 & -14 & -15 \\
\hline SBI & $\begin{array}{l}173 . \\
01\end{array}$ & $\begin{array}{l}196 . \\
00\end{array}$ & $\begin{array}{l}210 . \\
56\end{array}$ & $\begin{array}{l}243 . \\
08\end{array}$ & $\begin{array}{l}299 . \\
23\end{array}$ & $\begin{array}{l}357 . \\
00\end{array}$ & $\begin{array}{l}456 . \\
00\end{array}$ & $\begin{array}{l}556 . \\
00\end{array}$ & $\begin{array}{l}636 . \\
00\end{array}$ & $\begin{array}{l}704 . \\
70\end{array}$ & $\begin{array}{l}798 . \\
40\end{array}$ & $\begin{array}{l}943 . \\
90\end{array}$ & $\begin{array}{l}1064 \\
.00\end{array}$ & NA \\
\hline PNB & $\begin{array}{l}167 . \\
76\end{array}$ & $\begin{array}{l}195 . \\
64\end{array}$ & $\begin{array}{l}228 . \\
22\end{array}$ & $\begin{array}{l}276 . \\
88\end{array}$ & $\begin{array}{l}330 . \\
92\end{array}$ & $\begin{array}{l}407 . \\
41\end{array}$ & $\begin{array}{l}504 . \\
52\end{array}$ & $\begin{array}{l}654 . \\
92\end{array}$ & $\begin{array}{l}808 . \\
00\end{array}$ & $\begin{array}{l}1017 \\
.80\end{array}$ & $\begin{array}{l}1132 \\
.00\end{array}$ & $\begin{array}{l}1165 \\
.10\end{array}$ & $\begin{array}{l}1283 \\
.00\end{array}$ & $\begin{array}{l}1319 \\
.00\end{array}$ \\
\hline BOB & $\begin{array}{l}222 . \\
76\end{array}$ & $\begin{array}{l}237 . \\
67\end{array}$ & $\begin{array}{l}252 . \\
51\end{array}$ & $\begin{array}{l}310 . \\
36\end{array}$ & $\begin{array}{l}396 . \\
00\end{array}$ & $\begin{array}{l}555 . \\
00\end{array}$ & $\begin{array}{l}710 . \\
00\end{array}$ & $\begin{array}{l}914 . \\
00\end{array}$ & $\begin{array}{l}981 . \\
00\end{array}$ & $\begin{array}{l}1229 \\
.00\end{array}$ & $\begin{array}{l}1466 \\
.00\end{array}$ & $\begin{array}{l}1689 \\
.00\end{array}$ & $\begin{array}{l}1865 \\
.00\end{array}$ & $\begin{array}{l}1889 \\
.00\end{array}$ \\
\hline $\begin{array}{l}\text { Canar } \\
\text { a Bank }\end{array}$ & $\begin{array}{l}214 . \\
88\end{array}$ & $\begin{array}{l}250 . \\
11\end{array}$ & $\begin{array}{l}297 . \\
58\end{array}$ & $\begin{array}{l}351 . \\
12\end{array}$ & $\begin{array}{l}441 . \\
57\end{array}$ & $\begin{array}{l}548 . \\
76\end{array}$ & $\begin{array}{l}609 . \\
41\end{array}$ & $\begin{array}{l}780 . \\
17\end{array}$ & $\begin{array}{l}982 . \\
60\end{array}$ & $\begin{array}{l}1199 \\
.10\end{array}$ & $\begin{array}{l}1374 \\
.40\end{array}$ & $\begin{array}{l}1420 \\
.20\end{array}$ & $\begin{array}{l}1438 \\
.00\end{array}$ & $\begin{array}{l}1435 \\
.00\end{array}$ \\
\hline $\begin{array}{l}\text { Bank } \\
\text { of } \\
\text { India }\end{array}$ & $\begin{array}{l}218 . \\
74\end{array}$ & $\begin{array}{l}242 . \\
97\end{array}$ & $\begin{array}{l}266 . \\
72\end{array}$ & $\begin{array}{l}320 . \\
00\end{array}$ & $\begin{array}{l}381 . \\
00\end{array}$ & $\begin{array}{l}498 . \\
00\end{array}$ & $\begin{array}{l}652 . \\
00\end{array}$ & $\begin{array}{l}833 . \\
00\end{array}$ & $\begin{array}{l}1011 \\
.00\end{array}$ & $\begin{array}{l}1284 \\
.00\end{array}$ & $\begin{array}{l}1360 \\
.00\end{array}$ & $\begin{array}{l}1582 \\
.00\end{array}$ & $\begin{array}{l}1963 \\
.00\end{array}$ & $\begin{array}{l}2069 \\
.00\end{array}$ \\
\hline ICICI & $\begin{array}{l}486 . \\
49\end{array}$ & $\begin{array}{l}1120 \\
.00\end{array}$ & $\begin{array}{l}1010 \\
.00\end{array}$ & $\begin{array}{l}880 . \\
00\end{array}$ & $\begin{array}{l}905 . \\
00\end{array}$ & $\begin{array}{l}1027 \\
.00\end{array}$ & $\begin{array}{l}1008 \\
.00\end{array}$ & $\begin{array}{l}1154 \\
.00\end{array}$ & $\begin{array}{l}765 . \\
00\end{array}$ & $\begin{array}{l}735 . \\
00\end{array}$ & $\begin{array}{l}708 . \\
00\end{array}$ & $\begin{array}{l}735 . \\
00\end{array}$ & $\begin{array}{l}747 . \\
00\end{array}$ & $\begin{array}{l}832 . \\
00\end{array}$ \\
\hline HDFC & $\begin{array}{l}778 . \\
00\end{array}$ & $\begin{array}{l}865 . \\
00\end{array}$ & $\begin{array}{l}866 . \\
00\end{array}$ & $\begin{array}{l}806 . \\
00\end{array}$ & $\begin{array}{l}758 . \\
00\end{array}$ & $\begin{array}{l}607 . \\
00\end{array}$ & $\begin{array}{l}506 . \\
00\end{array}$ & $\begin{array}{l}446 . \\
00\end{array}$ & $\begin{array}{l}590 . \\
00\end{array}$ & $\begin{array}{l}653 . \\
00\end{array}$ & $\begin{array}{l}654 . \\
00\end{array}$ & $\begin{array}{l}750 . \\
00\end{array}$ & $\begin{array}{l}890 . \\
00\end{array}$ & $\begin{array}{l}1010 \\
.00\end{array}$ \\
\hline AXIS & $\begin{array}{l}896 . \\
00\end{array}$ & $\begin{array}{l}926 . \\
00\end{array}$ & $\begin{array}{l}808 . \\
00\end{array}$ & $\begin{array}{l}895 . \\
00\end{array}$ & $\begin{array}{l}1020 \\
.00\end{array}$ & $\begin{array}{l}1024 \\
.00\end{array}$ & $\begin{array}{l}1117 \\
.00\end{array}$ & $\begin{array}{l}1060 \\
.00\end{array}$ & $\begin{array}{l}1111 \\
.00\end{array}$ & $\begin{array}{l}1366 \\
.00\end{array}$ & $\begin{array}{l}1276 \\
.00\end{array}$ & $\begin{array}{l}1215 \\
.00\end{array}$ & $\begin{array}{l}1230 \\
.00\end{array}$ & $\begin{array}{l}1371 \\
.00\end{array}$ \\
\hline $\begin{array}{l}\text { Dhanl } \\
\text { axmi } \\
\text { Bank }\end{array}$ & $\begin{array}{l}199 . \\
24\end{array}$ & $\begin{array}{l}222 . \\
06\end{array}$ & $\begin{array}{l}248 . \\
63\end{array}$ & $\begin{array}{l}292 . \\
70\end{array}$ & $\begin{array}{l}311 . \\
71\end{array}$ & $\begin{array}{l}366 . \\
68\end{array}$ & $\begin{array}{l}409 . \\
08\end{array}$ & $\begin{array}{l}585 . \\
88\end{array}$ & $\begin{array}{l}369 . \\
60\end{array}$ & $\begin{array}{l}589 . \\
20\end{array}$ & $\begin{array}{l}592 . \\
90\end{array}$ & $\begin{array}{l}729 . \\
70\end{array}$ & $\begin{array}{l}774 . \\
00\end{array}$ & $\begin{array}{l}900 . \\
00\end{array}$ \\
\hline $\begin{array}{l}\text { Federa } \\
\text { l Bank }\end{array}$ & $\begin{array}{l}219 . \\
00\end{array}$ & $\begin{array}{l}270 . \\
00\end{array}$ & $\begin{array}{l}327 . \\
00\end{array}$ & $\begin{array}{l}366 . \\
00\end{array}$ & $\begin{array}{l}431 . \\
00\end{array}$ & $\begin{array}{l}544 . \\
00\end{array}$ & $\begin{array}{l}655 . \\
00\end{array}$ & $\begin{array}{l}750 . \\
00\end{array}$ & $\begin{array}{l}813 . \\
00\end{array}$ & $\begin{array}{l}923 . \\
00\end{array}$ & $\begin{array}{l}1011 \\
.00\end{array}$ & $\begin{array}{l}1075 \\
.00\end{array}$ & $\begin{array}{l}997 . \\
00\end{array}$ & $\begin{array}{l}1115 \\
.00\end{array}$ \\
\hline CITI & 1566 & 1660 & 1666 & 1359 & 1607 & 1360 & 1763 & 1880 & 1979 & 1745 & 1975 & 2124 & 2226 & 2463 \\
\hline Bank & .82 & .19 & .92 & .51 & .92 & .48 & .78 & .10 & .90 & .90 & .00 & .00 & .00 & .00 \\
\hline Abu & 2581 & 2618 & 1161 & 1061 & 1720 & 1865 & 1772 & 1759 & 1542 & 1607 & 1635 & 2718 & 3350 & 4026 \\
\hline Dhabi & .00 & .40 & .64 & .10 & .49 & .72 & .97 & .98 & .60 & .70 & .10 & .00 & .00 & .40 \\
\hline $\begin{array}{l}\text { Bank } \\
\text { of }\end{array}$ & $\begin{array}{l}1768 \\
.96\end{array}$ & $\begin{array}{l}1862 \\
.74\end{array}$ & $\begin{array}{l}1747 \\
.56\end{array}$ & $\begin{array}{l}1707 \\
.72\end{array}$ & $\begin{array}{l}1924 \\
.81\end{array}$ & $\begin{array}{l}1920 \\
.89\end{array}$ & $\begin{array}{l}2483 \\
.54\end{array}$ & $\begin{array}{l}2430 \\
.57\end{array}$ & $\begin{array}{l}3102 \\
.00\end{array}$ & $\begin{array}{l}3852 \\
.30\end{array}$ & $\begin{array}{l}3399 \\
.30\end{array}$ & $\begin{array}{l}3807 \\
.00\end{array}$ & $\begin{array}{l}4083 \\
.00\end{array}$ & $\begin{array}{l}4474 \\
.00\end{array}$ \\
\hline
\end{tabular}




\begin{tabular}{|c|c|c|c|c|c|c|c|c|c|c|c|c|c|c|}
\hline $\begin{array}{l}\text { Ameri } \\
\text { ca }\end{array}$ & & & & & & & & & & & & & & \\
\hline $\begin{array}{l}\text { Standa } \\
\text { rd } \\
\text { Charte } \\
\text { red } \\
\text { Bank }\end{array}$ & $\begin{array}{l}794 . \\
41\end{array}$ & $\begin{array}{l}840 . \\
54\end{array}$ & $\begin{array}{l}780 . \\
11\end{array}$ & $\begin{array}{l}786 . \\
36\end{array}$ & $\begin{array}{l}837 . \\
29\end{array}$ & $\begin{array}{l}924 . \\
20\end{array}$ & $\begin{array}{l}817 . \\
35\end{array}$ & $\begin{array}{l}971 . \\
77\end{array}$ & $\begin{array}{l}1083 \\
.50\end{array}$ & $\begin{array}{l}1345 \\
.60\end{array}$ & $\begin{array}{l}1546 \\
.70\end{array}$ & $\begin{array}{l}1687 \\
.70\end{array}$ & $\begin{array}{l}2071 \\
.00\end{array}$ & $\begin{array}{l}1900 \\
.00\end{array}$ \\
\hline HSBC & $\begin{array}{l}595 . \\
80 .\end{array}$ & $\begin{array}{l}622 . \\
78\end{array}$ & $\begin{array}{l}820 . \\
91 .\end{array}$ & $\begin{array}{l}779 . \\
45\end{array}$ & $\begin{array}{l}975 . \\
65\end{array}$ & $\begin{array}{l}979 . \\
68 .\end{array}$ & $\begin{array}{c}1012 \\
34\end{array}$ & $\begin{array}{l}961 . \\
81 .\end{array}$ & $\begin{array}{c}1135 \\
50\end{array}$ & $\begin{array}{c}1221 \\
70\end{array}$ & $\begin{array}{l}1658 \\
.90\end{array}$ & $\begin{array}{c}1889 \\
90\end{array}$ & $\begin{array}{l}2274 \\
.00\end{array}$ & $\begin{array}{l}2678 \\
.00\end{array}$ \\
\hline
\end{tabular}

(Source: RBI and IBA)

Business per employee measures the average business generated by each employee of a bank. In general, relatively high business per employee is a positive sign that suggests the bank is finding ways to squeeze more business out of each of its workers. The table no. 3 indicates that in Public sector banks business per employee is maximum in Bank of India, in private sector banks Axis Bank maintains the lead and in foreign sector banks Bank of America shows highest business per employee. But the percentage increase in last fourteen years is higher only in Public sector banks which show their improvement of performance.

Table 4:- Profit per employee of Banks selected for study (IN Rs. Lakhs)

\begin{tabular}{|c|c|c|c|c|c|c|c|c|c|c|c|c|c|c|}
\hline $\begin{array}{l}\text { Year/Ba } \\
\text { nk }\end{array}$ & $\begin{array}{l}200 \\
1- \\
02\end{array}$ & $\begin{array}{l}200 \\
2- \\
03\end{array}$ & $\begin{array}{l}200 \\
3- \\
04\end{array}$ & $\begin{array}{l}200 \\
4- \\
05\end{array}$ & $\begin{array}{l}200 \\
5- \\
06\end{array}$ & $\begin{array}{l}200 \\
6- \\
07\end{array}$ & $\begin{array}{l}2007 \\
-08\end{array}$ & $\begin{array}{l}2008 \\
-09\end{array}$ & $\begin{array}{l}2009 \\
-10\end{array}$ & $\begin{array}{l}2010 \\
-11\end{array}$ & $\begin{array}{l}2011 \\
-12\end{array}$ & $\begin{array}{l}2012 \\
-13\end{array}$ & $\begin{array}{l}2013 \\
-14\end{array}$ & $\begin{array}{l}2014 \\
-15\end{array}$ \\
\hline SBI & 1.16 & 1.47 & 1.77 & 2.08 & 2.17 & 2.37 & 3.73 & 4.74 & 4.50 & 3.90 & 5.30 & 6.50 & 4.85 & NA \\
\hline PNB & 0.97 & 1.43 & 1.88 & 2.42 & 2.48 & 2.68 & 3.66 & 5.64 & 7.30 & 8.40 & 8.40 & 8.10 & 5.00 & 5.00 \\
\hline BOB & 1.40 & 1.92 & 2.43 & 1.71 & 2.13 & 2.73 & 3.94 & 6.05 & 8.00 & $\begin{array}{l}11.0 \\
0\end{array}$ & $\begin{array}{l}12.0 \\
0\end{array}$ & $\begin{array}{l}10.0 \\
0\end{array}$ & 9.87 & 6.88 \\
\hline $\begin{array}{l}\text { Canara } \\
\text { Bank }\end{array}$ & 1.64 & 2.26 & 2.97 & 2.48 & 3.02 & 3.24 & 3.65 & 4.97 & 7.40 & 9.80 & 8.20 & 7.00 & 5.00 & 5.00 \\
\hline $\begin{array}{l}\text { Bank of } \\
\text { India }\end{array}$ & 1.16 & 1.97 & 2.35 & 0.80 & 1.66 & 2.71 & 4.95 & 7.49 & 4.40 & 6.20 & 6.40 & 6.40 & 10.00 & 7.00 \\
\hline ICICI & 5.33 & $\begin{array}{l}11.0 \\
0\end{array}$ & $\begin{array}{l}12.0 \\
0\end{array}$ & $\begin{array}{l}11.0 \\
0\end{array}$ & $\begin{array}{l}10.0 \\
0\end{array}$ & 9.00 & $\begin{array}{l}10.0 \\
0\end{array}$ & $\begin{array}{l}11.0 \\
0\end{array}$ & 9.00 & $\begin{array}{l}10.0 \\
0\end{array}$ & $\begin{array}{l}11.0 \\
0\end{array}$ & $\begin{array}{l}14.0 \\
0\end{array}$ & 14.00 & 16.00 \\
\hline HDFC & 9.75 & $\begin{array}{l}10.0 \\
9\end{array}$ & 9.39 & 8.80 & 7.39 & 6.13 & 4.97 & 4.18 & 6.00 & 7.40 & 8.00 & $\begin{array}{l}10.0 \\
0\end{array}$ & 12.00 & 10.00 \\
\hline AXIS & 7.79 & 8.22 & 8.07 & 8.02 & 8.69 & 7.59 & 8.39 & $\begin{array}{l}10.0 \\
2\end{array}$ & $\begin{array}{l}12.0 \\
0\end{array}$ & $\begin{array}{l}14.0 \\
0\end{array}$ & $\begin{array}{l}14.0 \\
0\end{array}$ & $\begin{array}{l}15.0 \\
0\end{array}$ & 15.42 & 17.07 \\
\hline $\begin{array}{l}\text { Dhanlax } \\
\text { mi Bank }\end{array}$ & 0.78 & 1.15 & 1.32 & $\begin{array}{l} \\
1.65\end{array}$ & 0.72 & 1.18 & 2.02 & 4.10 & 0.70 & 0.70 & -3.30 & 0.10 & $\begin{array}{l}(10.3 \\
7) \\
\end{array}$ & $\begin{array}{l}(10.6 \\
0) \\
\end{array}$ \\
\hline $\begin{array}{l}\text { Federal } \\
\text { Bank }\end{array}$ & 1.31 & 1.69 & 2.14 & 1.39 & 3.54 & 4.43 & 5.43 & 6.90 & 6.00 & 7.00 & 9.00 & 9.00 & 8.00 & 9.38 \\
\hline $\begin{array}{l}\text { CITI } \\
\text { Bank }\end{array}$ & $\begin{array}{l}22.1 \\
4\end{array}$ & $\begin{array}{l}24.2 \\
6\end{array}$ & $\begin{array}{l}28.3 \\
3\end{array}$ & $\begin{array}{l}21.7 \\
5\end{array}$ & $\begin{array}{l}21.7 \\
1\end{array}$ & $\begin{array}{l}17.3 \\
3\end{array}$ & $\begin{array}{l}37.7 \\
3\end{array}$ & $\begin{array}{l}45.1 \\
2\end{array}$ & $\begin{array}{l}18.3 \\
0\end{array}$ & $\begin{array}{l}28.6 \\
0\end{array}$ & $\begin{array}{l}36.0 \\
0\end{array}$ & $\begin{array}{l}50.2 \\
0\end{array}$ & 51.00 & 59.00 \\
\hline $\begin{array}{l}\text { Abu } \\
\text { Dhabi }\end{array}$ & $\begin{array}{l}11.0 \\
0\end{array}$ & 4.06 & $\begin{array}{l}12.2 \\
0\end{array}$ & $\begin{array}{l}- \\
77.2 \\
4\end{array}$ & $\begin{array}{l}12.0 \\
6\end{array}$ & 4.80 & $\begin{array}{l}78.2 \\
9\end{array}$ & $\begin{array}{l}41.9 \\
6\end{array}$ & $\begin{array}{l}18.6 \\
0\end{array}$ & $\begin{array}{l}19.1 \\
0\end{array}$ & $\begin{array}{l}34.0 \\
0\end{array}$ & $\begin{array}{l}35.7 \\
0\end{array}$ & N/A & N/A \\
\hline $\begin{array}{l}\text { Bank of } \\
\text { America }\end{array}$ & $\begin{array}{l}31.6 \\
7 \\
\end{array}$ & $\begin{array}{l}32.7 \\
6 \\
\end{array}$ & $\begin{array}{l}24.1 \\
2 \\
\end{array}$ & $\begin{array}{l}29.4 \\
7 \\
\end{array}$ & $\begin{array}{l}51.8 \\
2 \\
\end{array}$ & $\begin{array}{l}69.0 \\
9 \\
\end{array}$ & $\begin{array}{l}102 . \\
08\end{array}$ & $\begin{array}{l}110 . \\
85\end{array}$ & $\begin{array}{l}121 . \\
30\end{array}$ & $\begin{array}{l}139 . \\
00\end{array}$ & $\begin{array}{l}148 . \\
90\end{array}$ & $\begin{array}{l}120 . \\
70\end{array}$ & $\begin{array}{l}175.8 \\
7 \\
\end{array}$ & $\begin{array}{l}144.2 \\
5 \\
\end{array}$ \\
\hline $\begin{array}{l}\text { Standar } \\
\text { d } \\
\text { Chartere } \\
\text { d Bank }\end{array}$ & $\begin{array}{l}20.3 \\
8\end{array}$ & $\begin{array}{l}25.1 \\
5\end{array}$ & $\begin{array}{l}13.4 \\
0\end{array}$ & $\begin{array}{l}11.5 \\
0\end{array}$ & $\begin{array}{l}14.5 \\
0\end{array}$ & $\begin{array}{l}19.6 \\
2\end{array}$ & $\begin{array}{l}20.2 \\
2\end{array}$ & $\begin{array}{l}23.8 \\
2\end{array}$ & $\begin{array}{l}26.3 \\
0\end{array}$ & $\begin{array}{l}26.4 \\
0\end{array}$ & $\begin{array}{l}23.1 \\
0\end{array}$ & $\begin{array}{l}41.1 \\
0\end{array}$ & 23.87 & 41.79 \\
\hline HSBC & 4.97 & 4.50 & 6.32 & 8.90 & $\begin{array}{l}12.0 \\
7\end{array}$ & $\begin{array}{l}14.3 \\
2\end{array}$ & $\begin{array}{l}16.6 \\
9\end{array}$ & $\begin{array}{l}16.0 \\
6\end{array}$ & $\begin{array}{l}11.7 \\
0\end{array}$ & $\begin{array}{l}23.2 \\
0\end{array}$ & $\begin{array}{l}34.7 \\
0\end{array}$ & $\begin{array}{l}40.4 \\
0\end{array}$ & 31.03 & 34.25 \\
\hline
\end{tabular}

(Source: RBI and IBA) 
Profit per employee is a total profit of a bank divided by the number of employees. In general, the higher the number, the more efficient the bank uses its employees. In this study in public sector banks, Bank of India shows higher profit per employee. In private sector banks, Axis Bank shows highest ratio but Dhanlaxmi banks suffers loss. Yet in foreign sector banks, all the banks in good position but Bank of America shows higher percentage increase.

\section{Conclusion:-}

Performance of banks depends upon various factors. In this paper, performance is known by taking into account capital adequacy ratio, NPA, Profit per employee and Business per employee. In foreign sector banks, performance is better in terms of all these indicators as compare to other sector banks. NPA exists to the small extent in public and private sector bank but in some foreign sector banks it is near about zero which shows their sound business practices. In Capital adequacy ratio, only Dhanlaxmi bank shows declining trend yet others sector bank it is satisfactory.

\section{References:-}

1. Statistical reports of Banks in India

2. $\mathrm{RBI}-\mathrm{A}$ Profile of Banks in India

3. Published reports of Banks in India

4. Asian Journal of Research in Banking and Finance, Vol3. Issue 6 June 2013

5. A Working Paper No. 470, October, 2014 published by Indian Institute of Management, Bangalore)

6. Data Available in IBA. 